\title{
Uncertainty Inequalities on Laguerre hypergroup
}

\author{
Rahmouni Atef \\ University of Carthage, Faculty of Sciences of Bizerte \\ Department of Mathematics Bizerte 7021 Tunisia. \\ E-mail: Atef.Rahmouni@fsb.rnu.tn
}

\begin{abstract}
In this paper, we give analogues of local uncertainty inequality on $\mathbb{R}^{n}$ for stratified Laguerre hypergroup, connected with the spectral analysis of a given homogeneous sublaplacian $L$, also indicate how local uncertainty inequalities imply global uncertainty inequalities. It would be interesting to note that we deduce the local uncertainty inequalities for the radial functions on the Heisenberg group. Finally, we extend Heisenberg-Pauli-Weyl uncertainty inequality by ultracontractive properties of the semigroups generated by the differential operator and on the estimate on the heat kernel.
\end{abstract}

2000 Mathematics Subject Classification: 42B10, 42B30, 33C45.

Key words and phrases: Uncertainty principle, Heisenberg-Pauli-Weyl Inequality, Laguerre hypergroup, Laguerre Fourier transform.

\section{Introduction}

The serious question of certainty in science was high-lighted by Heisenberg, in 1927, via his uncertainty principle (cf. [16]). He demonstrated, for instance, the impossibility of specifying simultaneously the position and the speed (or the momentum) of an electron within an atom. In 1933, according to H. Hardy (cf. [14]).

$$
\text { A pair of transforms cannot both be very small. }
$$

This aspect of the uncertainty principle was already expounded by Norbert Wiener in a lecture in Göttingen in 1925. Unfortunately, no written record of this lecture seems to have survived, apart from the nontechnical account in Wiener's autobiography (cf. [34], pp. 105-107), so one can only guess at what precise versions of (1) it might have contained. Whatever influence this lecture might have had on the physicists in the audience, however, the uncertainty principle did not really sink into the minds of signal analysts until Gabor's fundamental work (cf. [11]) in 1946. Since then, it has become firmly embedded in the common culture.

On the mathematical side, there were sporadic developments relating to the uncertainty principle in the fifty years after the initial work in the 1920's, followed by a steady stream of results in the last tow decades, we refer to the survey article (cf. [10]) for an overview of the history, and the book (cf. [15]) of Havin and Jöricke for other forms of the uncertainty principle. These principles state that a function $f$ and its Fourier transform $\widehat{f}$ cannot be simultaneously sharply localized. The most common quantitative formulation of the uncertainty principle is the Heisenberg-Pauli-Weyl inequality. It says that, if $f \in L^{2}\left(\mathbb{R}^{n}\right)$

$$
\left(\int_{\mathbb{R}^{n}} x_{j}^{2}|f(x)|^{2} d x\right)\left(\int_{\mathbb{R}^{n}} \xi_{j}^{2}|\widehat{f}(\xi)|^{2} d \xi\right) \geq \frac{1}{4}\left(\int_{\mathbb{R}^{n}}|f(x)|^{2} d x\right)^{2}, j \in\{1,2, \ldots n\} .
$$

Recently, many works have been consecrated to establish the Heisenberg-Pauli-Weyl inequality for various Fourier transforms. Rösler ([26]) and Shimeno ([28]) have proved this inequality for the Dunkl transform, in ([27]) Rösler and Voit have established the analogue of Heisenberg Pauli-Weyl-inequality for the generalized Hankel transform. Also, in ([3]) De Bruijn, using the Hermite polynomials gave a new proof of the Heisenberg-Pauli-Weyl inequality for the classical Fourier transform.

An equivalent formulation of (2) is

$$
\left(\int_{\mathbb{R}^{n}}|x|^{2 \alpha}|f(x)|^{2} d x\right)\left(\int_{\mathbb{R}^{n}}\left|(-\Delta)^{\frac{\alpha}{2}} f(x)\right|^{2} d x\right) \geq C_{\alpha}\left(\int_{\mathbb{R}^{n}}|f(x)|^{2} d x\right)^{2},
$$


where $\Delta$ denotes the Laplacian on $\mathbb{R}^{n}$. This form of the Heisenberg-Pauli-Weyl inequality is better suited for extensions to other contexts, with the Laplacian replaced by a positive self-adjoint operator, and $|x|$ by a distance function. The interpretation of uncertainty inequalities as spectral properties of differential operators is widely present in the literature (cf. [8], [9], [31]).

In this paper we are interested in the Laguerre hypergroup $\mathbb{K}=[0,+\infty) \times \mathbb{R}$ which is the fundamental manifold of the radial function space for the Heisenberg group ([2], [18]). Let us recall that $\left(\mathbb{K}, *_{\alpha}\right)$ is a commutative hypergroup (cf. [20], [32] pp. 243-263), on which the involution and the Haar measure are respectively given by the homeomorphism $(x, t) \rightarrow(x, t)^{-}=(x,-t)$ and the Radon positive measure $d m_{\alpha}(x, t)=\frac{x^{2 \alpha+1}}{\pi \Gamma(\alpha+1)} d x d t$. The unity element of $\left(\mathbb{K}, *_{\alpha}\right)$ is given by $e=(0,0)$, i.e. $\delta_{(x, t)} *_{\alpha} \delta_{(0,0)}=\delta_{(0,0)} *_{\alpha}$ $\delta_{(x, t)}=\delta_{(x, t)}$ for all $(x, t) \in \mathbb{K}$. The convolution product $*_{\alpha}$ is defined for two bounded Radon measures $\mu$ and $v$ on $\mathbb{K}$ as follows

$$
\left\langle\mu *_{\alpha} v, f\right\rangle=\int_{\mathbb{K} \times \mathbb{K}} T_{(x, t)}^{(\alpha)} f(y, s) d \mu(x, t) d v(y, s),
$$

where $\alpha$ is a fixed nonnegative real number and $\left\{T_{(x, t)}^{(\alpha)}\right\}_{(x, t) \in \mathbb{K}}$ are the translation operators on the Laguerre hypergroup (cf. [2], [20], [29], [32]), given by

$$
T_{(x, t)}^{(\alpha)} f(y, s)=\left\langle\boldsymbol{\delta}_{(x, t)} * \alpha \boldsymbol{\delta}_{(y, s)}, f\right\rangle= \begin{cases}\frac{\alpha}{\pi} \int_{0}^{1} \int_{0}^{2 \pi} f\left((\xi, \eta)_{r, \theta}\right) r\left(1-r^{2}\right)^{\alpha-1} d \theta d r & \text { if } \alpha>0, \\ \frac{1}{2 \pi} \int_{0}^{2 \pi} f\left((\xi, \eta)_{1, \theta}\right) d \theta & \text { if } \alpha=0,\end{cases}
$$

where $(\xi, \eta)_{r, \theta}=\left(\sqrt{x^{2}+y^{2}+2 x y r \cos \theta}, t+s+x y r \sin \theta\right)$.

Note that for the particular case $\mu=f m_{\alpha}$ and $v=g m_{\alpha}, f$ and $g$ being two suitable functions on $\mathbb{K}$, one has $\mu *_{\alpha} v=\left(f *_{\alpha} g\right) m_{\alpha}$, where $f *_{\alpha} g$ is the convolution product of $f$ and $g$ given by

$$
f *_{\alpha} g(x, t)=\int_{\mathbb{K} \times \mathbb{K}} T_{(-y, s)}^{(\alpha)} f(x, t) g(y, s) d m_{\alpha}(x, t) .
$$

The dual (cf. p. 46, [2]) of Laguerre hypergroup, i.e. the space of all bounded continuous and multiplicative functions $\chi: \mathbb{K} \rightarrow \mathbb{C}$ such that such that $\widetilde{\chi}=\chi$, where $\widetilde{\chi}(x, t)=\overline{\chi(x,-t)},(x, t) \in \mathbb{K}$, is given ([21]) by $\widehat{\mathbb{K}}=\left\{\varphi_{\lambda, m} ;(\lambda, m) \in \mathbb{R}^{*} \times \mathbb{N}\right\} \cup\left\{\varphi_{\rho} ; \rho \geq 0\right\}$, where $\varphi_{\lambda, m}(x, t)=e^{-i \lambda t} \mathscr{L}_{m}^{(\alpha)}\left(|\lambda| x^{2}\right)$ and $\varphi_{\rho}=j_{\alpha}(\rho x) ; \mathscr{L}_{m}^{(\alpha)}(x)=e^{-\frac{x^{2}}{2}} L_{m}^{(\alpha)}(x) / L_{m}^{(\alpha)}(0)$ and $j_{\alpha}(x)=2^{\alpha} \Gamma(\alpha+1) \frac{J_{\alpha}(x)}{x^{\alpha}}, J_{\alpha}$ being the Bessel function of first kind and order $\alpha$, and $L_{m}^{(\alpha)}$ being the Laguerre polynomial of degree $m$ and order $\alpha$ (cf. [19], [30]). $\widehat{\mathbb{K}}$ which can be seen as a deformation of the hypergroup of radial functions on the Heisenberg group.

The dual of the Laguerre hypergroup $\widehat{\mathbb{K}}$ can be topologically identified with the so-called Heisenberg fan (cf. [6]), i.e., the subset embedded in $\mathbb{R}^{2}$ given by

$$
\left(\bigcup_{m \in \mathbb{N}}\left\{(\lambda, \mu) \in \mathbb{R}^{2}: \mu=|\lambda|(2 m+\alpha+1), \lambda \neq 0\right\}\right) \bigcup\left(\left\{(0, \mu) \in \mathbb{R}^{2}: \mu \geq 0\right\}\right) .
$$

Moreover, the subset $\left\{(0, \mu) \in \mathbb{R}^{2} ; \mu \geq 0\right\}$ has zero Plancherel measure; therefore it will usually be disregarded. Identifying $\widehat{\mathbb{K}}$ and $\left(\mathbb{R}^{*} \times \mathbb{N}\right) \cup[0,+\infty[$, the Fourier transform of a bounded Radon measure $\mu$ on the Laguerre hypergroup is then, by

$$
\mathscr{F}(\mu)(\lambda, m)=\int_{\mathbb{K}} \varphi_{-\lambda, m}(x, t) d \mu(x, t) \text { and } \mathscr{F}(\mu)(\rho)=\int_{\mathbb{K}} j_{\alpha}(\rho, x) d \mu(x, t) .
$$

The Fourier Laguerre transform of a suitable function $f: \mathbb{K} \rightarrow \mathbb{C}$ is given by $\widehat{f}=\mathscr{F}\left(f d m_{\alpha}\right)$, so that

$$
\widehat{f}(\lambda, m)=\int_{\mathbb{K}} f(x, t) \varphi_{-\lambda, m}(x, t) d m_{\alpha}(x, t) \text { and } \widehat{f}(\rho)=\int_{\mathbb{K}} f(x, t) j_{\alpha}(\rho x) d m_{\alpha}(x, t) .
$$

The functional analysis and Fourier analysis on $\mathbb{K}$ and its dual $\widehat{\mathbb{K}}$ have been extensively studied in (cf. [1], [20]), and hence it is well known that the Fourier Laguerre transform given above is a topological 
isomorphism from the Schwartz space on $\mathbb{K}$ onto $S(\widehat{\mathbb{K}})$ : the Schwartz space on $\widehat{\mathbb{K}}$. Its inverse is the operator $f^{\vee}$ given by

$$
f^{\vee}(x, t)=\int_{\mathbb{R} \times \mathbb{N}} \varphi_{\lambda, m}(x, t) f d \gamma_{\alpha},
$$

where $d \gamma_{\alpha}$ is the Plancherel measure on $\widehat{\mathbb{K}}$ given by $d \gamma_{\alpha}(\lambda, m)=L_{m}^{\alpha}(0) \delta_{m} \otimes|\lambda|^{\alpha+1} d \lambda$.

We introduce the following notations (cf. [20]) $L_{\alpha}^{p}(\mathbb{K})\left(\right.$ resp. $L_{\alpha}^{p}(\mathbb{R} \times \mathbb{N})$ ) where $1 \leq p \leq \infty$ the p-th Lebesgue space on $\mathbb{K}$ (resp. on $\mathbb{R} \times \mathbb{N}$ ) formed by the measurable functions $f: \mathbb{K} \rightarrow \mathbb{C}$ (resp. $\Phi: \mathbb{R} \times \mathbb{N} \rightarrow \mathbb{C}$ ) such that $\|f\|_{L_{\alpha}^{p}(\mathbb{K})}<+\infty\left(\right.$ resp. $\left.\|f\|_{L_{\alpha}^{p}(\mathbb{R} \times \mathbb{N})}<+\infty\right)$ where

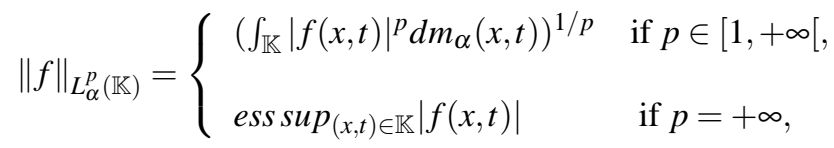

and

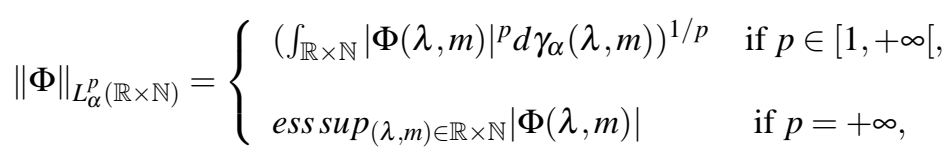

we have the following Plancherel formula

$$
\|f\|_{L_{\alpha}^{2}(\mathbb{K})}=\|\widehat{f}\|_{L_{\alpha}^{2}(\widehat{\mathbb{K}})}, \quad f \in L_{\alpha}^{1}(\widehat{\mathbb{K}}) \cap L_{\alpha}^{2}(\widehat{\mathbb{K}})
$$

and we have

$$
\|\widehat{f}\|_{L_{\alpha}^{\infty}} \leq\|f\|_{L_{\alpha}^{1}}
$$

This paper is organized as follows.

In section 2 we set some notations and collect some basic facts about the Laguerre hypergroup. In section 3 of central interest is the following slight sharpening of a local uncertainty inequality. Heisenberg's inequality says that $f$ is highly localized, then $\widehat{f}$ can not be concentrated near a single point, but it does not preclude $\widehat{f}$ from being concentrated in a small neighborhood of two or more widely separated points. In fact, the latter phenomenon cannot occur either, and it is the object of local uncertainty inequality to make this precise. The first such inequalities for the Fourier transform were obtained by Faris (cf. [7]), and they were subsequently sharpened and generalized by Price (cf. [22], [23]). Building on the ideas of Faris, Price, and Ricci (cf. [5]) we show the inequalities of the uncertainty on the local Laguerre hypergroup, it is the subject of the following results.

(a) If $0<\beta<\frac{2 \alpha+4}{2}$, there is a constant $K=K(\alpha, \beta)$ such that for every $f \in L_{\alpha}^{2}(\mathbb{K})$, and every measurable set $E \subset \widehat{\mathbb{K}} ; \quad 0<\gamma_{\alpha}(E)<\infty$,

$$
\int_{\widehat{\mathbb{K}}}|\widehat{f}(\lambda, m)|^{2} \chi_{E} d \gamma_{\alpha}(\lambda, m) \leq K \gamma_{\alpha}(E)^{\frac{\beta}{2 \alpha+4}}\left\||(x, t)|^{\beta} f\right\|_{2}^{2} .
$$

(b) If $\beta>\frac{2 \alpha+4}{2}$, there is a constant $\widetilde{K}=\widetilde{K}(\alpha, \beta)$ such that for every $f \in L_{\alpha}^{2}(\mathbb{K})$, and every measurable set $E \subset \widehat{\mathbb{K}} ; 0<\gamma_{\alpha}(E)<\infty$,

$$
\int_{\widehat{\mathbb{K}}}|\widehat{f}(\lambda, m)|^{2} \chi_{E} d \gamma_{\alpha} \leq \widetilde{K} \gamma_{\alpha}(E)\|f\|_{2}^{2-\frac{2 \alpha+4}{\beta}}\left\||(x, t)|^{\beta} f\right\|_{2}^{\frac{2 \alpha+4}{\beta}} .
$$

(c) Cases (a) and (b) fail for $\beta=\frac{2 \alpha+4}{2}$.

We also indicate in the proof of the Corollary 1 how local uncertainty inequalities imply global uncertainty inequalities. It would be interesting to note that we deduce the local uncertainty inequalities for the radial functions on the Heisenberg group. 
Finally (Section 4), building on the ideas of (cf. [4]) to establish an inequality analogous to (3), and variants of it, for the Laguerre hypergroup. Our purpose is to prove a general form of the HeisenbergPauli-Weyl inequality in Laguerre hypergroup. More precisely, using the spectral theory, ultracontractive properties of the semigroups generated by partial differential operator and the estimate on the heat kernel on Laguerre hypergroup, that is for all $f \in L_{\alpha}^{2}(\mathbb{K}), a, b \in \mathbb{R} ; a, b \geq 1$ and $\eta \in \mathbb{R}$ such that $\eta a=(1-\eta) b$, we have

$$
\left(\int_{\mathbb{K}}|(x, t)|^{2 a}|f(x, t)|^{2} d m_{\alpha}(x, t)\right)^{\frac{\eta}{2}}\left(\int_{\mathbb{R} \times \mathbb{N}}|(\lambda, m)|^{b / 2}|\widehat{f}(\lambda, m)|^{2} d \gamma_{\alpha}(\lambda, m)\right)^{\frac{1-\eta}{2}} \geq C\|f\|_{2} .
$$

Throughout this paper, $C$ will always represent a positive constant, not necessarily the same in each occurrence.

\section{Preliminaries}

To describe the harmonic analysis in our setting we begin with introducing the operator

$$
L=-\left(\frac{\partial^{2}}{\partial x^{2}}+\frac{2 \alpha+1}{x} \frac{\partial}{\partial x}+x^{2} \frac{\partial^{2}}{\partial t^{2}}\right)
$$

which is positive, symmetric in $L_{\alpha}^{2}(\mathbb{K})$, we endow the space $\mathbb{K}$ with homogeneous of degree one norm (with respect to the family dilations $\left(\delta_{\rho}\right)_{\rho>0}$ ),

$$
N(x, t)=|(x, t)|=\left(x^{4}+4 t^{2}\right)^{1 / 4}, \quad(x, t) \in \mathbb{K} .
$$

For $\alpha=n-1, n$ being a positive integer, the operator is the radial part of the sub-Laplacian on the Heisenberg group $\mathbb{H}^{n}$ (cf. [29]).

Also, we introduce the operator $\Lambda=\left(\Lambda_{1}^{2}-\left(2 \Lambda_{2}+2 \frac{\partial}{\partial \lambda}\right)^{2}\right)$ defined on $\widehat{\mathbb{K}}$, where $\Lambda=\frac{1}{|\lambda|}\left(m \Delta_{+} \Delta_{-}+\right.$ $\left.(\alpha+1) \Delta_{+}\right)$and $\Lambda=\frac{-1}{2|\lambda|}\left((m+\alpha+1) \Delta_{+}+m \Delta_{-}\right)$.

$\Delta \pm$ are given for a suitable function $\Phi$ by: $\Delta_{+} \Phi(\lambda, m)=\Phi(\lambda, m+1)-\Phi(\lambda, m), \Delta_{-} \Phi(\lambda, m)=\Phi(\lambda, m)-$ $\Phi(\lambda, m-1)$, if $m \geq 1$ and $\Delta_{-} \Phi(\lambda, 0)=\Phi(\lambda, 0)$, and the quasinorm

$$
\mathscr{N}(\lambda, m)=|(\lambda, m)|=4|\lambda|\left(m+\frac{\alpha+1}{2}\right), \quad(\lambda, m) \in \widehat{\mathbb{K}} .
$$

These operators satisfy some basic properties which can be found in (cf. [1], [20], [21]) namely one has $L \varphi_{\lambda, m}=-|(\lambda, m)| \varphi_{\lambda, m}$ and $\Lambda \varphi_{\lambda, m}=|(x, t)|^{4} \varphi_{\lambda, m}$, by the properties of $L$ and the symmetry one can observe that $\widehat{L f}(\lambda, m)=4|\lambda|\left(m+\frac{\alpha+1}{2}\right) \widehat{f}(\lambda, m)$ (cf. [29]), and we define the following operator

$$
\widehat{L^{b / 2} f}(\lambda, m)=|(\lambda, m)|^{b / 2} \widehat{f}(\lambda, m), \quad(\lambda, m) \in \widehat{\mathbb{K}} .
$$

We will denote by $\delta_{\rho}(x, t)=\left(\rho x, \rho^{2} t\right)$, the dilated of $(x, t) \in \mathbb{K}$ and by $B_{r}(x, t)$ the ball centered at $(x, t)$ of radius $r$, i.e., the set $B_{r}(x, t)=\left\{(y, s) \in \mathbb{K}:|(x-y, t-s)|_{\mathbb{K}}<r\right\}$, and $\omega_{\alpha}=\frac{\Gamma\left(\frac{\alpha+1}{2}\right)}{2 \sqrt{\pi} \Gamma\left(\frac{\alpha}{2}+1\right) \Gamma(\alpha+1)}$ its surface area of $B_{r}=B_{r}(0,0)$ (cf. [12]). Denote by

$$
f_{\rho}(x, t)=\rho^{(2 \alpha+4)} f\left(\delta_{\frac{1}{\rho}}(x, t)\right)
$$

the dilated of the function $f$ defined on $\mathbb{K}$ preserving the mean of $f$ with respect to the measure $d m_{\alpha}$, in the sense that

$$
\left\|f_{\rho}\right\|_{L_{\alpha}^{1}(\mathbb{K})}=\|f\|_{L_{\alpha}^{1}(\mathbb{K})}, \quad \forall f \in L_{\alpha}^{1}(\mathbb{K}), \rho>0 .
$$

Throughout this paper, let us fix the notation of the norm by $\|-\|_{L_{\alpha}^{p}(\mathbb{K})}=\|-\|_{p}, \quad \forall p \geq 1$. 


\section{The Local Uncertainty Principle}

The purpose of this section we develop a family of inequalities in their sharpest forms, which constitute the principle of local uncertainty. It is the subject of the following theorem.

\section{Theorem 1.}

(a) If $0<\beta<\frac{2 \alpha+4}{2}$, there is a constant $K=K(\alpha, \beta)$ such that for every $f \in L_{\alpha}^{2}(\mathbb{K})$, and every measurable set $E \subset \widehat{\mathbb{K}} ; 0<\gamma_{\alpha}(E)<\infty$,

$$
\int_{\widehat{\mathbb{K}}}|\widehat{f}(\lambda, m)|^{2} \chi_{E} d \gamma_{\alpha}(\lambda, m) \leq K \gamma_{\alpha}(E)^{\frac{\beta}{2 \alpha+4}}\left\||(x, t)|^{\beta} f\right\|_{2}^{2},
$$

where

$$
K(\alpha, \beta)=\left(\frac{\alpha+2}{\alpha+2-\beta}\right)\left(\frac{\omega_{\alpha}}{\beta^{2}}\right)^{\frac{\beta}{2 \alpha+4}}
$$

(b) If $\beta>\frac{2 \alpha+4}{2}$, there is a constant $\widetilde{K}=\widetilde{K}(\alpha, \beta)$ such that for every $f \in L_{\alpha}^{2}(\mathbb{K})$, and every measurable set $E \subset \widehat{\mathbb{K}} ; 0<\gamma_{\alpha}(E)<\infty$,

$$
\int_{\widehat{\mathbb{K}}}|\widehat{f}(\lambda, m)|^{2} \chi_{E} d \gamma_{\alpha} \leq \widetilde{K} \gamma_{\alpha}(E)\|f\|_{2}^{2-\frac{2 \alpha+4}{\beta}}\left\||(x, t)|^{\beta} f\right\|_{2}^{\frac{2 \alpha+4}{\beta}},
$$

where

$$
\widetilde{K}(\alpha, \beta)=\frac{\pi \omega_{\alpha}}{(\beta-(\alpha+2)) \sin \left(\frac{(\alpha+2) \pi}{\beta}\right)}\left(\frac{\alpha+2}{\beta-(\alpha+2)}\right)^{-\frac{\alpha+2}{\beta-(\alpha+2)}} .
$$

(c) Cases (a) and (b) fail for $\beta=\frac{2 \alpha+4}{2}$.

Proof. Let $B_{r}$ denote the closed unit ball in $\mathbb{K}$ and $B_{r}^{c}$ its complement. Denote by $\chi_{B_{r}}$ and $\chi_{B_{r}^{c}}$ the characteristic functions.

Part(a), let $f \in L_{\alpha}^{2}(\mathbb{K})$. By Minkowski's inequality and (4), for all $r>0$, we have

$$
\begin{aligned}
\left\|\widehat{f} \chi_{E}\right\|_{2} & \leq\left\|\left(f \chi_{B_{r}}\right) \hat{\chi_{E}}\right\|_{2}+\left\|\left(f \chi_{B_{r}^{c}}\right) \widehat{ } \chi_{E}\right\|_{2} \\
& \leq\left(\gamma_{\alpha}(E)\right)^{1 / 2}\left\|\left(f \chi_{B_{r}}\right)\right\|_{\infty}+\left\|\left(f \chi_{B_{r}^{c}}\right) \chi_{E}\right\|_{2} \\
& \leq\left(\gamma_{\alpha}(E)\right)^{1 / 2}\left\|f \chi_{B_{r}}\right\|_{1}+\left\|\left(f \chi_{B_{r}^{c}}\right)\right\|_{2} .
\end{aligned}
$$

On the other hand, by Hölder's inequality and hypothesis $\beta<\frac{2 \alpha+4}{2}$, we have

$$
\begin{aligned}
\left\|f \chi_{B_{r}}\right\|_{1} & \leq\left\||(x, t)|^{-\beta} \chi_{B_{r}}\right\|_{2}\left\||(x, t)|^{\beta} f\right\|_{2} \\
& \leq\left(\frac{\frac{\Gamma\left(\frac{\alpha+1}{2}\right)}{2 \sqrt{\pi} \Gamma\left(\frac{\alpha}{2}+1\right) \Gamma(\alpha+1)}}{\alpha+2-\beta}\right)^{1 / 2} r^{\alpha+2-\beta}\left\||(x, t)|^{\beta} f\right\|_{2}, \\
& \leq\left(\frac{\omega_{\alpha}}{\alpha+2-\beta}\right)^{1 / 2} r^{\alpha+2-\beta}\left\||(x, t)|^{\beta} f\right\|_{2} .
\end{aligned}
$$

By Plancherel's theorem, we have

$$
\begin{aligned}
\|\left(f \chi_{B_{r}^{c}} \widehat{)} \|_{2}\right. & =\left\|f \chi_{B_{r}^{c}}\right\|_{2} \\
& \leq\left\||(x, t)|^{-\beta} \chi_{B_{r}^{c}}\right\|_{\infty}\left\||(x, t)|^{\beta} f\right\|_{2} \\
& \leq r^{-\beta}\left\||(x, t)|^{\beta} f\right\|_{2} .
\end{aligned}
$$


Combining the relations (8), (9) and (10), by choosing $r_{0}>0$ to satisfy

$$
r_{0}^{2 \alpha+4}=\left(\frac{\beta^{2}}{\gamma_{\alpha}(E) \omega_{\alpha}}\right)
$$

we minimize the quantity on the right side to obtain

$$
\left(\int_{\widehat{\mathbb{K}}}|\widehat{f}(\lambda, m)|^{2} \chi_{E} d \gamma_{\alpha}\right)^{1 / 2} \leq K(\alpha, \beta)\left(\gamma_{\alpha}(E)\right)^{\frac{\beta}{2 \alpha+4}}\left\||(x, t)|^{\beta} f\right\|_{2},
$$

where

$$
K(\alpha, \beta)=\left(\frac{\alpha+2}{\alpha+2-\beta}\right)\left(\frac{\omega_{\alpha}}{\beta^{2}}\right)^{\frac{\beta}{2 \alpha+4}} .
$$

Part (b), from the hypothesis $\beta>\frac{2 \alpha+4}{2}$, we deduce that the function

$$
(x, t) \mapsto\left(1+|(x, t)|^{2 \beta}\right)^{-1}
$$

belongs to $L_{\alpha}^{1}(\mathbb{K}) \cap L_{\alpha}^{2}(\mathbb{K})$ and by Hölder's inequality, we have

$$
\begin{aligned}
\|f\|_{1}^{2} & \leq\left\|\left(1+|(x, t)|^{2 \beta}\right)^{1 / 2} f\right\|_{2}^{2}\left\|\left(1+|(x, t)|^{2 \beta}\right)^{-1 / 2}\right\|_{2}^{2} \\
& \leq\left(\|f\|_{2}^{2}+\left\||(x, t)|^{2 \beta} f\right\|_{2}^{2}\right)\left\|\left(1+|(x, t)|^{2 \beta}\right)^{-1 / 2}\right\|_{2}^{2} .
\end{aligned}
$$

However, by standard calculus and (cf. [13], page 322), we have

$$
\left\|\left(1+|(x, t)|^{2 \beta}\right)^{-1 / 2}\right\|_{2}^{2}=\frac{\pi \omega_{\alpha}}{\beta \sin \left(\frac{(\alpha+2) \pi}{\beta}\right)} .
$$

For $\rho>0$, we put as above $f_{\rho}(x, t)=\rho^{-(2 \alpha+4)} f\left(\frac{x}{\rho}, \frac{t}{\rho^{2}}\right)$, then we have

$$
\left\|f_{\rho}\right\|_{2}^{2}=\rho^{-(2 \alpha+4)}\|f\|_{2}^{2}
$$

and

$$
\left\||(x, t)|^{\beta} f_{\rho}\right\|_{2}^{2}=\rho^{2 \beta-(2 \alpha+4)}\left\||(x, t)|^{\beta} f\right\|_{2}^{2} .
$$

Replacing $f$ by $f_{\rho}$ in the relation (11), and by equation (12) we deduce

$$
\|f\|_{1}^{2} \leq \frac{\pi \omega_{\alpha}}{\beta \sin \left(\frac{(\alpha+2) \pi}{\beta}\right)}\left(\rho^{-2(\alpha+2)}\|f\|_{2}^{2}+\rho^{2(\beta-(\alpha+2))}\left\||(x, t)|^{\beta} f\right\|_{2}^{2}\right) .
$$

In particular for

$$
\rho_{0}^{2 \beta}=\left(\frac{\alpha+2}{\beta-(\alpha+2)} \frac{\|f\|_{2}^{2}}{\left\||(x, t)|^{\beta} f\right\|_{2}^{2}}\right)
$$

we get

$$
\|f\|_{1}^{2} \leq \frac{\pi \omega_{\alpha}}{(\beta-(\alpha+2)) \beta \sin \left(\frac{(\alpha+2) \pi}{\beta}\right)}\left(\frac{\alpha+2}{\beta-(\alpha+2)}\right)^{-\frac{\alpha+2}{\beta-(\alpha+2)}}\|f\|_{2}^{2-\frac{2 \alpha+4}{\beta}}\left\||(x, t)|^{\beta} f\right\|_{2}^{\frac{2 \alpha+4}{\beta}} .
$$

Now, according the relations (13), the function $f \in L_{\alpha}^{1}(\mathbb{K})$ we have

$$
\begin{aligned}
\int_{\widehat{\mathbb{K}}}|\widehat{f}(\lambda, m)|^{2} \chi_{E} d \gamma_{\alpha} & \leq \gamma_{\alpha}(E)\|\widehat{f}\|_{\infty}^{2} \\
& \leq \gamma_{\alpha}(E)\|f\|_{1}^{2} \\
& \leq \widetilde{K}(\alpha, \beta) \gamma_{\alpha}(E)\|f\|_{2}^{2-\frac{2 \alpha+4}{\beta}}\left\||(x, t)|^{\beta} f\right\|_{2}^{\frac{2 \alpha+4}{\beta}}
\end{aligned}
$$


where

$$
\widetilde{K}(\alpha, \beta)=\frac{\pi \omega_{\alpha}}{(\beta-(\alpha+2)) \sin \left(\frac{(\alpha+2) \pi}{\beta}\right)}\left(\frac{\alpha+2}{\beta-(\alpha+2)}\right)^{-\frac{\alpha+2}{\beta-(\alpha+2)}} .
$$

Part (c), we collect together the counterexamples necessary to establish Theorem 1.

Counterexample1: Necessary to establish Theorem 1(a). We consider the function $f_{1}(x, t)=h_{1}(t) g_{1}(x)$ with

$$
h_{1}(t)=\left\{\begin{array}{ll}
|t|^{-1 / 4} & \text { if }|t| \leq 1, \\
0 & \text { elsewhere }
\end{array} \quad \text { and } \quad g_{1}(x)= \begin{cases}|x|^{2\left(\frac{3}{2} \beta-\frac{3}{2}-\alpha\right)} & \text { if }|x| \leq 1 \\
0 & \text { elsewhere }\end{cases}\right.
$$

where $\frac{2 \alpha+4}{2} \geq \beta>\frac{2 \alpha+4}{6}$.

The function $f_{1} \in L_{\alpha}^{2}(\mathbb{K})$, we shall prove that if $\beta=\frac{2 \alpha+4}{2}$ then $\left\|\widehat{f}_{1}(\lambda, m) \chi_{E}\right\|_{2}^{2}=+\infty$. Let $C_{m}=\frac{1}{\pi \Gamma(\alpha+1) L_{m}^{\alpha}(0)}$. Then we have

$$
\widehat{f}_{1}(\lambda, m)=\frac{C_{m}}{|\lambda|^{\frac{1}{2}\left(3 \beta+\frac{1}{2}\right)}}\left(\int_{0}^{|\lambda|} u^{-1 / 4} \cos (u) d u\right)\left(\int_{0}^{|\lambda|} e^{-u / 2} L_{m}^{\alpha}(u) u^{\frac{3}{2}(\beta-1)} d u\right) .
$$

Take $E=B_{R}(0,0)($ cf. Lemma 2$)$ and as $L_{0}^{\alpha}(0)=1$. We have

$$
\begin{aligned}
\left\|\widehat{f}_{1}(\lambda, m) \chi_{E}\right\|_{2}^{2} & =\sum_{m=0}^{\infty} L_{m}^{\alpha}(0) \int_{\mathbb{R}}\left|\widehat{f}_{1}(\lambda, m) \chi_{E}\right|^{2}|\lambda|^{\alpha+1} d \lambda . \\
& =\sum_{m=0}^{\infty} L_{m}^{\alpha}(0) \int_{\frac{-R}{m+\frac{\alpha+1}{2}}}^{\frac{R}{m+\frac{\alpha+1}{2}}}\left|\widehat{f}_{1}(\lambda, m)\right|^{2}|\lambda|^{\alpha+1} d \lambda . \\
& \geq \frac{2}{[\pi \Gamma(\alpha+1)]^{2}} \int_{0}^{\frac{\alpha}{2}}\left|\widetilde{g}_{1}(\lambda)\right|^{2}|\lambda|^{\alpha-3 \beta+\frac{1}{2}} d \lambda,
\end{aligned}
$$

where $\widetilde{g}_{1}(\lambda)=\left(\int_{0}^{|\lambda|} u^{-1 / 4} \cos (u) d u\right)\left(\int_{0}^{|\lambda|} e^{-u / 2} u^{\frac{3}{2}(\beta-1)} d u\right)$.

But we have $\int_{0}^{\infty} u^{-1 / 4} \cos (u) d u=\Gamma\left(\frac{3}{4}\right) \cos \left(\frac{3 \pi}{8}\right)$ and $\int_{0}^{\infty} e^{-u / 2} u^{\frac{3}{2}(\beta-1)} d u=2^{\frac{3}{2} \beta-\frac{1}{2}} \Gamma\left(\frac{3 \beta}{2}-\frac{1}{2}\right)$. So, there exist $C>0, R>0$, and $r>0$, such that for $R \geq|\lambda| \geq r$ we have $\left|\widetilde{g}_{1}(\lambda)\right| \geq C$ and $\left\|\widehat{f}_{1}(\lambda, m) \chi_{E}\right\|_{2}^{2} \geq$ $C \int_{r}^{\frac{\alpha+1}{2}}|\lambda|^{\alpha-3 \beta+\frac{1}{2}} d \lambda \rightarrow+\infty$ as $r \rightarrow 0^{+}$, if $\frac{\alpha}{3}+\frac{1}{2}<\beta$. In particular for $\beta=\frac{2 \alpha+4}{2}$, this provides a contradiction since the left side of (5) is bounded. Indeed, by (8) we have,

$$
\left\|\widehat{f}_{1} \chi_{E}\right\|_{2} \leq\left(\gamma_{\alpha}(E)\right)^{1 / 2}\left\|f_{1} \chi_{B_{r}}\right\|_{1}+\|\left(f_{1} \chi_{B_{r}^{c}} \widehat{)} \|_{2} .\right.
$$

So, $\|\left(f_{1} \chi_{B_{r}^{c}} \widehat{)} \|_{2} \leq \frac{r^{3 \beta+1 / 2}}{4 \beta+1 / 2} B\left(\frac{3}{8}, \frac{\beta+2 \alpha-1}{4}\right)\right.$ and $\left\|f_{1} \chi_{B_{r}}\right\|_{1}=\frac{r^{3 \beta+1 / 2}}{3 \beta+1 / 2} B\left(\frac{3}{8}, \frac{3 \beta-1}{4}\right)$, whereas $\left\|\widehat{f}_{1} \chi_{E}\right\|_{2} \leq\left(\frac{B\left(\frac{3}{8}, \frac{\beta+2 \alpha-1}{4}\right)}{4 \beta+1 / 2}+\right.$ $\left.\frac{B\left(\frac{3}{8}, \frac{3 \beta-1}{4}\right)}{3 \beta+1 / 2}\right) r^{3 \beta+1 / 2} \longrightarrow 0$ as $r \longrightarrow 0^{+}$.

Counterexample 2: Necessary to establish Theorem 1(b). We consider the function $f_{2}(x, t)=h_{1}(t) g_{2}(x)$ with

$$
h_{1}(t)=\left\{\begin{array}{ll}
|t|^{-1 / 4} & \text { if }|t| \leq 1, \\
0 & \text { elsewhere }
\end{array} \quad \text { and } \quad g_{2}(x)= \begin{cases}|x|^{2\left(\frac{\beta}{2}-\frac{5}{4}-\alpha\right)} & \text { if }|x| \leq 1 \\
0 & \text { elsewhere }\end{cases}\right.
$$

where $\beta \geq \frac{2 \alpha+4}{2}>\frac{2 \alpha+3}{2}$. 
The function $f_{2} \in L_{\alpha}^{2}(\mathbb{K})$, we shall prove that if $\beta=\frac{2 \alpha+4}{2}$ then $\left\|\widehat{f}_{2}(\lambda, m) \chi_{E}\right\|_{2}^{2}=+\infty$. Let $C_{m}=\frac{1}{\pi \Gamma(\alpha+1) L_{m}^{\alpha}(0)}$. Then we have

$$
\widehat{f_{2}}(\lambda, m)=\frac{C_{m}}{|\lambda|^{\frac{\beta-1}{2}}}\left(\int_{0}^{|\lambda|} u^{-1 / 4} \cos (u) d u\right)\left(\int_{0}^{|\lambda|} e^{-u / 2} L_{m}^{\alpha}(u) u^{\frac{\beta}{2}-\frac{5}{2}} d u\right) .
$$

Take $E=B_{R}(0,0)$ (cf. Lemma 2) and as $L_{0}^{\alpha}(0)=1$. We have

$$
\begin{aligned}
\left\|\widehat{f}_{2}(\lambda, m) \chi_{E}\right\|_{2}^{2} & =\sum_{m=0}^{\infty} L_{m}^{\alpha}(0) \int_{\mathbb{R}}\left|\widehat{f}_{2}(\lambda, m) \chi_{E}\right|^{2}|\lambda|^{\alpha+1} d \lambda \\
& =\sum_{m=0}^{\infty} L_{m}^{\alpha}(0) \int_{\frac{-R}{m+\frac{\alpha+1}{2}}}^{\frac{R}{m+\frac{\alpha+1}{2}}}\left|\widehat{f}_{2}(\lambda, m)\right|^{2}|\lambda|^{\alpha+1} d \lambda \\
& \geq \frac{2}{[\pi \Gamma(\alpha+1)]^{2}} \int_{1}^{\frac{\alpha}{2}}\left|\widetilde{g}_{2}(\lambda)\right|^{2}|\lambda|^{\alpha-\beta+2} d \lambda
\end{aligned}
$$

where $\widetilde{g}_{2}(\lambda)=\left(\int_{1}^{|\lambda|} u^{-1 / 4} \cos (u) d u\right)\left(\int_{1}^{|\lambda|} e^{-u / 2} u^{\frac{\beta}{2}-\frac{5}{2}} d u\right)$.

But we have $\int_{0}^{\infty} u^{-1 / 4} \cos (u) d u=\Gamma\left(\frac{3}{4}\right) \cos \left(\frac{3 \pi}{8}\right)$ and $\int_{0}^{\infty} e^{-u / 2} u^{\frac{\beta}{2}-\frac{5}{2}} d u=2^{\frac{\beta}{2}-\frac{3}{2}} \Gamma\left(\frac{\beta}{2}-\frac{3}{2}\right)$. So, there exist $C>0, R>1$, such that for $R \geq|\lambda| \geq 1$ we have $\left|\widetilde{g}_{2}(\lambda)\right| \geq C$ and $\left\|\widehat{f}_{2}(\lambda, m) \chi_{E}\right\|_{2}^{2} \geq C \int_{1}^{\frac{\frac{R}{\alpha+1}}{2}}|\lambda|^{\alpha-\beta+2} d \lambda \rightarrow$ $+\infty$ as $R \rightarrow \infty$, if $\frac{2 \alpha+6}{2}>\beta$. In particular for $\beta=\frac{2 \alpha+4}{2}$, this provides a contradiction since the left side of (7) is bounded. Indeed, by (14) we have,

$$
\left\|\widehat{f}_{1} \chi_{E}\right\|_{2} \leq \gamma_{\alpha}(E)\left\|f_{1}\right\|_{1} \leq \frac{2 C}{2 \beta+3} B\left(\frac{3}{8}, \frac{\beta}{4}-\frac{1}{8}\right) .
$$

\section{Remarks 1.}

1. The relations among the exponents in these inequalities are forced by homogeneity considerations $Q=2 \alpha+4$ of Laguerre hypergroup $\mathbb{K}$.

2. Price and Sitaram ([24], Remark 5.2) proved the following local uncertainty inequality on the Heisenberg group $H^{n}$ : Given $0<\theta<\frac{1}{2}$, for each $f \in L^{1}\left(\mathbb{H}^{n}\right) \cap L^{2}\left(\mathbb{H}^{n}\right)$ and $E$ with Lebesgue measure $m(E)<\infty$,

$$
\left(\int_{E} \operatorname{Tr}\left(\pi_{\lambda}(f)^{*} \pi_{\lambda}(f)\right) d \mu(\lambda)\right)^{1 / 2} \leq K_{\theta} m(E)^{\theta}\left\||\cdot|^{\theta} f\right\|_{2}
$$

where

$$
K_{\theta}=\left(2 \theta^{2}\right)^{-\theta}(1-2 \theta)^{-1}(1-2 \theta)^{\theta} .
$$

Using Theorem 1a), let $\theta=\frac{\beta}{2 \alpha+4}$ which satisfies $0<\theta<\frac{1}{2}$. For each $f \in L_{\text {rad }}^{1}\left(\mathbb{H}^{n}\right) \cap L_{\text {rad }}^{2}\left(\mathbb{H}^{n}\right)$, we can replace their $K_{\theta}$ by the sharper one

$$
K_{\theta, \text { rad }}(n-1, \beta)=\left(2 \theta^{2}\right)^{-\theta}(1-2 \theta)^{-1}\left(\frac{\omega_{n-1}}{2(n+1)^{2}}\right)^{\theta},
$$

and $\omega_{n-1}=\frac{\Gamma\left(\frac{n}{2}\right)}{2 \sqrt{\pi} \Gamma\left(\frac{n+1}{2}\right) \Gamma(n)}$.

3. It would be interesting to know if this is the best possible. 
We shall use the local uncertainty inequality (5) to prove an analogue of Heisenberg-Pauli-Weyl uncertainty inequality, therefore we are so led to impose the most important lemma that we need for the sequel.

For this purpose we define the ball in $\widehat{\mathbb{K}}$ with center $(\lambda, m)$ and radius $r>0$ ( for shortness $B_{r}$ ) to be the set

$$
B_{r}=\left\{(\mu, n) \in \widehat{\mathbb{K}}, \mathscr{N}\left(\lambda-\mu, \max (n-m, 0)+\frac{\alpha+1}{2}\right)<r\right\} .
$$

Examples: Figs of two balls with center $(0,0)$ and $(2,6)$ respectively and radius $r=1$.

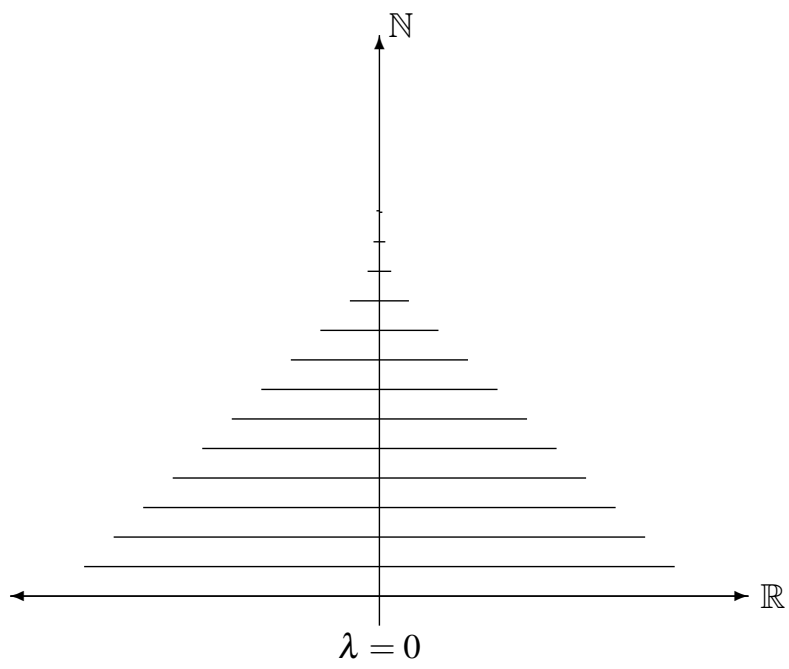

Fig 1: Ball $B_{1}(0,0)$

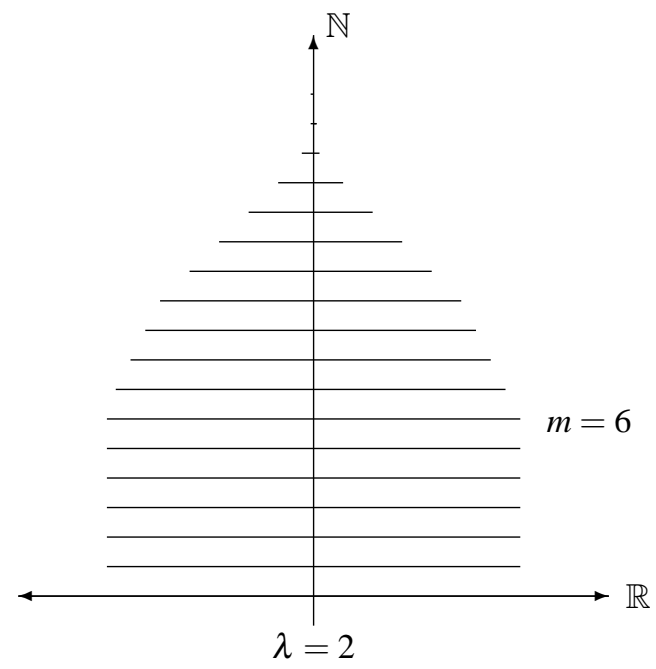

Fig 2: Ball $B_{1}(2,6)$

Lemma 1. The measure of $B_{r}$ with respect to the Plancherel measure $d \gamma_{\alpha}$ is finite, in the sense that for all $r>0$ and $(\lambda, m) \in \widehat{\mathbb{K}}$

$$
\gamma_{\alpha}\left(B_{r}\right)<\infty
$$

Proof. For fixed $(\lambda, m) \in \widehat{\mathbb{K}}$ and $r>0$ one has

$$
\begin{aligned}
\gamma_{\alpha}\left(B_{r}\right) & =\int_{B_{r}} d \gamma_{\alpha}(\mu, n) \\
& =\sum_{n=0}^{\infty} L_{n}^{\alpha}(0) \int_{\frac{m a x}{\max (n-m, 0)+\frac{\alpha+1}{2}}+\lambda}^{\frac{r}{2}+\lambda}|\mu|^{\alpha+1} d \mu \\
& =\sum_{n=m+1}^{\infty} L_{n}^{\alpha}(0) \int_{\frac{r-r+\frac{\alpha+1}{2}}{n-m+\frac{\alpha+1}{2}}+\lambda}^{\frac{n+m}{2}}|\mu|^{\alpha+1} d \mu+\sum_{n=0}^{m} L_{n}^{\alpha}(0) \int_{\frac{-r}{\frac{\alpha+1}{2}}+\lambda}^{\frac{r}{\frac{\alpha+1}{2}}+\lambda}|\mu|^{\alpha+1} d \mu \\
& =I_{1}+I_{2}
\end{aligned}
$$

where

$$
I_{1}=\frac{1}{\alpha+2} \sum_{n=m+1}^{\infty} L_{n}^{\alpha}(0)\left[\left(\frac{r}{n-m+\frac{\alpha+1}{2}}+\lambda\right)^{\alpha+2}-\left(-\frac{r}{n-m+\frac{\alpha+1}{2}}+\lambda\right)^{\alpha+2}\right]
$$

and

$$
I_{2}=\frac{\left[\left(\frac{r}{\frac{\alpha+1}{2}}+\lambda\right)^{\alpha+2}-\left(-\frac{r}{\frac{\alpha+1}{2}}+\lambda\right)^{\alpha+2}\right]}{\alpha+2} \sum_{n=0}^{m} L_{n}^{\alpha}(0) .
$$


Since $L_{n}^{\alpha}(0) \sim \frac{n^{\alpha}}{\Gamma(\alpha+1)}$, it follows $\frac{L_{n}^{\alpha}(0)}{\alpha+2}\left[\left(\frac{r}{n+\frac{\alpha+1}{2}}+\lambda\right)^{\alpha+2}-\left(\frac{r}{n+\frac{\alpha+1}{2}}-\lambda\right)^{\alpha+2}\right] \sim \frac{C_{r}}{n^{2}}$, and that $d \gamma_{\alpha}\left(B_{r}\right)<\infty$, where we have computed the result above for the case $\mathscr{N}(\lambda, m)<r$ for all $m \geq 0$. An analogous result follows for the complement case.

Remark 1. The volume of the ball $B_{r}$ depends not only on its radius $r$, but also it is largely close to its center $(\lambda, m)$ which means that the Plancherel measure $d \gamma_{\alpha}$ is not invariant under the standard translation over $\mathbb{R}^{2}$.

Throughout subsequently, we take the measurable set in $\widehat{\mathbb{K}}$ by

$$
E_{r}=\left\{(\lambda, m) \in \widehat{\mathbb{K}},|(\lambda, m)|=4|\lambda|\left(m+\frac{\alpha+1}{2}\right)<r^{2}\right\},
$$

that is the ball in $\widehat{\mathbb{K}}$ with center $(0,0)$ and radius $r^{2}$.

Lemma 2. The measure of $E_{r}$ with respect to the Plancherel measure $d \gamma_{\alpha}$ is finite, in the sense that for all $r>0$

$$
\gamma_{\alpha}\left(E_{r}\right)<\infty
$$

More precisely,

$$
\gamma_{\alpha}\left(E_{r}\right)=2 \frac{r^{2(\alpha+2)}}{\alpha+2} \sum_{m \geq 0} \frac{L_{m}^{\alpha}(0)}{\left(m+\frac{\alpha+1}{2}\right)^{\alpha+2}}
$$

Proof. For fixed $r>0$ one has

$$
\begin{aligned}
\gamma_{\alpha}\left(E_{r}\right) & =\int_{E_{r}} d \gamma_{\alpha}(\lambda, m) \\
& =\sum_{m \geq 0} L_{m}^{\alpha}(0) \int_{\frac{-r^{2}}{m+\frac{\alpha+1}{2}}}^{\frac{r^{2}}{m+\frac{\alpha+1}{2}}}|\lambda|^{\alpha+1} d \lambda \\
& =2 \frac{r^{2(\alpha+2)}}{\alpha+2} \sum_{m \geq 0} \frac{L_{m}^{\alpha}(0)}{\left(m+\frac{\alpha+1}{2}\right)^{\alpha+2}} .
\end{aligned}
$$

One can remark easily that the volume of the set $E_{r}$ is finite since $L_{m}^{\alpha}(0) \sim \frac{m^{\alpha}}{\Gamma(\alpha+1)}$, so $\frac{L_{m}^{\alpha}(0)}{\left(m+\frac{\alpha+1}{2}\right)^{\alpha+2}} \sim \frac{C}{m^{2}}$ and hence $\gamma_{\alpha}\left(E_{r}\right)<\infty$.

Corollary 1. For all $f \in L_{\alpha}^{2}(\mathbb{K})$ and $\beta>0$ one has

$$
\left(\int_{\mathbb{K}}|(x, t)|^{2 \beta}|f(x, t)|^{2} d m_{\alpha}(x, t)\right)\left(\int_{\widehat{\mathbb{K}}}|(\lambda, m)|^{\beta / 2}|\widehat{f}(\lambda, m)|^{2} d \gamma_{\alpha}(\lambda, m)\right) \geq C\|f\|_{2}^{4},
$$

where $C$ is a constant.

Proof. First, suppose that $0<\beta<\frac{2 \alpha+4}{2}$.

Let $E_{r}$ be the subset of $\widehat{\mathbb{K}}$ and $E_{r}^{c}$ denotes the complement of $E_{r}$ in $\widehat{\mathbb{K}}$. Then, by Plancherel's theorem, we have

$$
\begin{aligned}
\|f\|_{2}^{2} & =\int|\widehat{f}(\lambda, m)|^{2} d \gamma_{\alpha}(\lambda, m) \\
& =\int_{E_{r}}|\widehat{f}(\lambda, m)|^{2} d \gamma_{\alpha}+\int_{E_{r}^{c}}|\widehat{f}(\lambda, m)|^{2} d \gamma_{\alpha}
\end{aligned}
$$


Consider

$$
\begin{aligned}
\int_{E_{r}^{c}}|\widehat{f}(\lambda, m)|^{2} d \gamma_{\alpha} & =\int_{E_{r}^{c}}|\widehat{f}(\lambda, m)|^{2}|(\lambda, m)|^{\beta}|(\lambda, m)|^{-\beta} d \gamma_{\alpha} \\
& \leq\left.\left. r^{-2 \beta} \int_{E_{r}^{c}}||(\lambda, m)\right|^{\beta / 2}|\widehat{f}(\lambda, m)|\right|^{2} d \gamma_{\alpha} \\
& \leq C r^{-2 \beta} \int_{\widehat{\mathbb{K}}}\left|L^{\beta / 2 f(\lambda, m)}\right|^{2} d \gamma_{\alpha} .
\end{aligned}
$$

By the local uncertainty inequality (a), with $0<\frac{\beta}{2 \alpha+4}<\frac{1}{2}$, we get

$$
\int_{E_{r}}|\widehat{f}(\lambda, m)|^{2} d \gamma_{\alpha} \leq C r^{2 \beta} \int_{\mathbb{K}}|(x, t)|^{2 \beta}|f(x, t)|^{2} d m_{\alpha}
$$

Combining the relations (17), (18) and (19), we obtained

$$
\|f\|_{2}^{2} \leq C\left(r^{2 \beta} \int_{\mathbb{K}}|(x, t)|^{2 \beta}|f(x, t)|^{2} d m_{\alpha}+r^{-2 \beta} \int_{\widehat{\mathbb{K}}}\left|L^{\beta / 2 f(\lambda, m)}\right|^{2} d \gamma_{\alpha}\right) .
$$

However, let $g$ be the function defined on $] 0,+\infty[$ by

$$
g(r)=r^{2 \beta}\left\||(x, t)|^{\beta} f\right\|_{2}^{2}+r^{-2 \beta}\left\||(\lambda, m)|^{\beta / 2} \widehat{f}\right\|_{2}^{2},
$$

then, the minimum of the function $\mathrm{g}$ is attained of the point

$$
r_{0}=\left(\frac{\left\||(\lambda, m)|^{\beta / 2} \widehat{f}\right\|_{2}^{2}}{\left\||(x, t)|^{\beta} f\right\|_{2}^{2}}\right)^{\frac{1}{4 \beta}}
$$

and

$$
g\left(r_{0}\right)=2\left\||(x, t)|^{\beta} f\right\|_{2}\left\||(\lambda, m)|^{\beta / 2} \widehat{f}\right\|_{2} .
$$

Then we have the desired inequality

$$
\|f\|_{2}^{4} \leq C\left(\int_{\mathbb{K}}|(x, t)|^{2 \beta}|f(x, t)|^{2} d m_{\alpha}(x, t)\right)\left(\int_{\widehat{\mathbb{K}}}\left|L^{\beta / 2} \widehat{f^{2}(\lambda, m)}\right|^{2} d \gamma_{\alpha}(\lambda, m)\right) .
$$

Second, for $\beta \geq \frac{2 \alpha+4}{2}$. By Hölder's inequality, we have

$$
\int_{\mathbb{K}}|f(x, t)|^{2}|(x, t)|^{2 \beta} d m_{\alpha}(x, t) \leq\|f\|_{2}\left(\int_{\mathbb{K}}|f(x, t)|^{2}|(x, t)|^{4 \beta} d m_{\alpha}(x, t)\right)^{1 / 2}
$$

and

$$
\begin{aligned}
\int_{\widehat{\mathbb{K}}}\left|L^{\beta / 2} f(\lambda, m)\right|^{2} d \gamma_{\alpha} & \leq \int_{\widehat{\mathbb{K}}}|(\lambda, m)|^{\beta}|\widehat{f}(\lambda, m)||\widehat{f}(\lambda, m)| d \gamma_{\alpha} \\
& \leq\left(\int_{\widehat{\mathbb{K}}}|\widehat{f}(\lambda, m)|^{2} d \gamma_{\alpha}\right)^{1 / 2}\left(\int_{\widehat{\mathbb{K}}}\left|L^{\beta} f(\lambda, m)\right|^{2} d \gamma_{\alpha}\right)^{1 / 2} \\
& \leq\|f\|_{2}\left(\int_{\widehat{\mathbb{K}}}\left|L^{\beta} f(\lambda, m)\right|^{2} d \gamma_{\alpha}\right)^{1 / 2}
\end{aligned}
$$

the value of in (20) can be replaced by $2 \beta$, and doing it repeatedly, (20) is extended to any positive $\beta$. 


\section{Heisenberg-Pauli-Weyl inequality}

In this section, we extend the inequality of the uncertainty of Heisenberg-Pauli-Weyl (Corollary 1) to the more general case. We need to use another method based on ultracontractive semigroups generated by the differential operator $L$ and the estimation of the heat kernel, the result gives by

Theorem 2. Let $a, b \geq 1$ and $\eta \in \mathbb{R}$ such that $\eta a=(1-\eta) b$, then for all $f \in L_{\alpha}^{2}(\mathbb{K})$, we have

$$
\left(\int_{\mathbb{K}}|(x, t)|^{2 a}|f(x, t)|^{2} d m_{\alpha}(x, t)\right)^{\frac{\eta}{2}}\left(\left.\int_{\widehat{\mathbb{K}}}|(\lambda, m)|^{b / 2} \widehat{f}(\lambda, m)\right|^{2} d \gamma_{\alpha}(\lambda, m)\right)^{\frac{1-\eta}{2}} \geq C_{\alpha}\|f\|_{2}
$$

where $C_{\alpha}$ is a constant.

Remark 2. In the particular case when $a=b=\beta$ and $\eta=\frac{1}{2}$, the previous result gives us the HeisenbergPauli-Weyl inequality ( cf. Corollary 1)

$$
\left\||(x, t)|^{2 \beta} f\right\|_{2}^{2}\left\||(\lambda, m)|^{\beta / 2} \widehat{f}\right\|_{2}^{2} \geq C\|f\|_{2}^{4} .
$$

In our proof of Theorem 2, the heat kernel $h_{s}$ plays an important role.

\subsection{Characterization of the heat kernel.}

The heat kernel on $\mathbb{K}$ is an analogue of the Gauss kernel $p_{s}$ on $\mathbb{R}^{n}$, there is associated to the operator $L$. Let $\left\{H^{s}: s>0\right\}=\left\{e^{-s L}: s>0\right\}$ defines a semigroup (heat-diffusion semigroup) of operators such that for any $\phi \in C_{0}^{\infty}(\mathbb{K}), H^{s} \phi$ is a solution of

$$
L_{(x, t)}+\frac{\partial}{\partial s}=0
$$

and $H^{s} \phi \longrightarrow \phi$ a.e. as $s \longrightarrow 0$. For every $s>0, H_{s}$ is an integral operator with kernel $h_{s}$, i.e. for any $\phi \in C_{0}^{\infty}(\mathbb{K})$,

$$
H^{s} \phi=\phi * h_{s} .
$$

Then $h_{s}, s>0$ are bi-invariant functions and $h$ as a function of the variables $s \in \mathbb{R}_{+}$satisfying the properties (cf. [29]).

Proposition 1. The heat kernel $h_{s}$ satisfies that

(1) $\left\{h_{s}: s>0\right\}$ form a semigroup under convolution $*$. That is, $h_{s} * h_{t}=h_{s+t}$ for $s, t>0$,

(2) $h_{s}(x,-t)=h_{s}(x, t), \quad \int_{\mathbb{K}} h_{s}(x, t) d m_{\alpha}(x, t)=1$.

(3) $h_{s}$ is a fundamental solution of $\left(L+\frac{\partial}{\partial s}\right) h_{s}=0$, on $\mathbb{K} \times(0,+\infty)$.

(4) $h_{\rho^{2} s}\left(\rho s, \rho^{2} t\right)=\rho^{-(2 \alpha+4)} h_{s}(x, t)$.

Furthermore,

$$
\widehat{h}_{s}(\lambda, m)=e^{-4|\lambda|\left(m+\frac{\alpha+1}{2}\right) s} .
$$

The pointwise estimate of the heat kernel $h_{s}(x, t)$ is given by (cf. [17]).

Lemma 3. There are positive constants $C$ and $A$ such that

$$
h_{s}(x, t)=\int_{\mathbb{R}}\left(\frac{\lambda}{2 \sinh (2 \lambda s)}\right)^{\alpha+1} e^{-\frac{1}{2} \lambda \operatorname{coth}(2 \lambda s) x^{2}} e^{i \lambda t} d \lambda,
$$

and

$$
h_{s}(x, t) \leq C s^{-(\alpha+2)} e^{-\frac{A}{s}|(x, t)|^{2}} .
$$


Lemma 4. Let $a>0$. Then for all $f \in L_{\alpha}^{2}(\mathbb{K})$, we have

$$
\left\|e^{-s L} f\right\|_{2} \leq C s^{-a / 2}\left\||(x, t)|^{a} f\right\|_{2}
$$

where $C$ is a constant.

Proof. For $r>0$, let denote the characteristic function of $\{(x, t):|(x, t)|<r\}$.

We set $f_{r}=f \chi_{B_{r}}$, and $f^{r}=f-f_{r}$. Then, since $\left|f^{r}(x, t)\right| \leq r^{-a}|(x, t)|^{a} f(x, t) \mid$ and $e^{-s L}$ is a semigroup of contractions,

$$
\left\|e^{-s L} f^{r}\right\|_{2} \leq\left\|f^{r}\right\|_{2} \leq r^{-a}\left\||(x, t)|^{a} f\right\|_{2}
$$

On the other hand, we have

$$
\begin{aligned}
\left\|e^{-s L} f_{r}\right\|_{2}=\left\|f_{r} * h_{s}\right\|_{2} \leq & \left\|f_{r}\right\|_{1}\left\|h_{s}\right\|_{2} \\
\leq & \left\|h_{s}\right\|_{2}\left(\int_{\mathbb{K}}|(x, t)|^{2 a}\left|f_{r}(x, t)\right|^{2} d m_{\alpha}(x, t)\right)^{1 / 2} \\
& \left(\int_{\mathbb{K}}|(x, t)|^{-2 a} \chi_{B_{r}} d m_{\alpha}(x, t)\right)^{1 / 2}
\end{aligned}
$$

since,

$$
\int_{\mathbb{K}}|(x, t)|^{-2 a} \chi_{B_{r}} d m_{\alpha}(x, t)=\omega_{\alpha} r^{(2 \alpha+4)-2 a},
$$

which gives,

$$
\left\|e^{-s L} f_{r}\right\|_{2} \leq C r^{-a+(\alpha+2)}\left\||(x, t)|^{a} f\right\|_{2}\left\|h_{s}\right\|_{2} .
$$

By (23), we have

$$
\left\|h_{s}\right\|_{2} \leq C s^{-\frac{(\alpha+1)}{2}} \text {. }
$$

Hence,

$$
\begin{aligned}
\left\|e^{-s L} f\right\|_{2} & \leq\left\|e^{-s L} f_{r}\right\|_{2}+\left\|e^{-s L} f^{r}\right\|_{2} \\
& \leq C r^{-a}\left(1+r^{(\alpha+2)} s^{-\frac{(\alpha+1)}{2}}\right)\left\||(x, t)|^{a} f\right\|_{2} .
\end{aligned}
$$

Choosing $r=s^{1 / 2}$, we obtain (24).

\subsection{Proof of Theorem 2.}

Let $f \in L_{\alpha}^{2}(\mathbb{K})$ satisfy the hypothesis. For all $s>0$, by Lemma 4 and spectral Theorem we have

$$
\begin{aligned}
\|f\|_{2} & \leq\left\|e^{-s L} f\right\|_{2}+\left\|\left(1-e^{-s L}\right) f\right\|_{2} \\
& \leq C\left(s^{-a / 2}\left\||(x, t)|^{a} f\right\|_{2}+\left\|\left(1-e^{-s L}\right)(s L)^{-b / 2}(s L)^{b / 2} f\right\|_{2}\right) .
\end{aligned}
$$

The last term is controlled, using spectral Theorem (Theorem VIII.5 p.262, [25]) by $s^{b / 2}\left\|L^{b / 2} f\right\|_{2}$ since $\left(1-e^{-s}\right) s^{-b / 2}$ is bounded for $s \geq 0$ if $b \leq 2$. Hence, we obtain

$$
\|f\|_{2} \leq C\left(s^{-a / 2}\left\|e^{-s L} f\right\|_{2}+s^{b / 2}\left\|L^{b / 2} f\right\|_{2}\right) .
$$

Then we obtain,

$$
\|f\|_{2} \leq C\left(s^{-a / 2}\left\||(x, t)|^{a} f\right\|_{2}+s^{b / 2}\left\||(\lambda, m)|^{b / 2} \widehat{f}(\lambda, m)\right\|_{2}\right) .
$$


However, let $g$ be the function defined on $] 0,+\infty[$ by

$$
g(s)=s^{-a / 2}\left\||(x, t)|^{a} f\right\|_{2}+s^{b / 2}\left\||(\lambda, m)|^{b / 2} \widehat{f}\right\|_{2},
$$

then, the minimum of the function $g$ is attained of the point

$$
s_{0}=\left(\frac{a}{b} \frac{\left\||(x, t)|^{a} f\right\|_{2}}{\left\||(\lambda, m)|^{b / 2} \widehat{f}\right\|_{2}}\right)^{\frac{2}{a+b}}
$$

and

$$
g\left(s_{0}\right)=\left(\frac{a}{b}\right)^{2 \eta-1}\left\||(x, t)|^{a} f\right\|_{2}^{\eta}\left\||(\lambda, m)|^{b / 2} \widehat{f}\right\|_{2}^{1-\eta} .
$$

From which optimizing in $s$, we obtain the result

$$
\|f\|_{2} \leq C\left(\left\||(x, t)|^{a} f\right\|_{2}^{\eta}\left\||(\lambda, m)|^{b / 2} \widehat{f}\right\|_{2}^{1-\eta}\right),
$$

for $b \leq 2$.

If $b>2$, let $b^{\prime} \leq 2$. For $u \geq 0$ and $b^{\prime}<b, u^{b^{\prime}} \leq 1+u^{b}$ which for $u=\left(\frac{|(\lambda, m)|}{\varepsilon}\right)^{1 / 2}$ gives the inequality $\left(\frac{|(\lambda, m)|}{\varepsilon}\right)^{b^{\prime} / 2} \leq 1+\left(\frac{|(\lambda, m)|}{\varepsilon}\right)^{b / 2}$, for all $\varepsilon>0$. It follows that

$$
\left\||(\lambda, m)|^{b^{\prime} / 2} \widehat{f}\right\|_{2} \leq \varepsilon^{b^{\prime} / 2}\|f\|_{2}+\varepsilon^{\frac{b^{\prime}-b}{2}}\left\||(\lambda, m)|^{b / 2} \widehat{f}\right\|_{2} .
$$

Let

$$
g(\varepsilon)=\varepsilon^{b^{\prime} / 2}\|f\|_{2}+\varepsilon^{\frac{b^{\prime}-b}{2}}\left\||(\lambda, m)|^{b / 2} \widehat{f}\right\|_{2}
$$

then, the minimum of the function $g$ is attained of the point

$$
\varepsilon_{0}=\left(\frac{b-b^{\prime}}{b^{\prime}} \frac{\left\||(\lambda, m)|^{b / 2} \widehat{f}\right\|_{2}}{\|f\|_{2}}\right)^{2 / b}
$$

Optimizing in $\varepsilon_{0}$, we get $\left\||(\lambda, m)|^{b^{\prime} / 2} \widehat{f}\right\|_{2} \leq C\|f\|_{2}^{1-b^{\prime} / b}\left\||(\lambda, m)|^{b / 2} \widehat{f}\right\|_{2}^{b^{\prime} / b}$. Plugging this into (21) with is replaced by $b$, we get the result for $b>2$.

Acknowledgements. I want to express my sincere gratitude to Professor Gerald B. Folland for useful discussions which have influenced some parts of the text for detecting and removing some misprints. Also, I would like to thank Professor Burglind Juhl-Jöricke for his advice, and his general remarks.

\section{References}

[1] M. Assal, Pseudo-differential operators associated with Laguerre hypergroups. J. Comp. Appl. Math., 233 (2009), 617-620.

[2] W. Bloom, H. Heyer, Harmonic Analysis of Probability Measures on Hypergroups. De Gruyter Stud. Math., vol. 20, Berlin/New York, 1994.

[3] N. G. De Bruijn, Uncertainty principles in Fourier analysis. Inequalities (Proc. Sympos. Wright-Patterson Air Force Base, Ohio, 1965), Academic Press, New York, (1967), 57-71.

[4] P. Ciatti, F. Ricci, M. Sundari, Heisenberg-Pauli-Weyl uncertainty inequalities and polynomial volume growth. Adv. Math., 215 (2007), 616-625.

[5] P. Ciatti, F. Ricci, M. Sundari, A local uncertainty inequality for step-two nilpotent Lie groups. In: Proc. Internat. Conf. in Harmonic Analysis and Quantum Groups, Bull. Kerala Math. Ass. 3 (2006), 53-72.

[6] J. Faraut, K. Harzallah, Deux cours d'Analyse Harmonique. In: Ecole d'été d'Analyse Harmonique de Tunis, Birkhaüser, 1984. 
[7] W. G. Faris, Inequalities and uncertainty inequalities, J. Math. Phys., 19 (1978), 461-466.

[8] C. Fefferman, D. H. Phong, The uncertainty principle and sharp Gårding inequality. Comm. Pure Appl. Math., 34 (1981), 285-331.

[9] C. Fefferman, The uncertainty principle. Bull. Amer. Math. Soc., 9 (1983), 129-206.

[10] G. Folland, A. Sitaram, The uncertainty principle: A mathematical survey. J. Fourier An. Appl., 3 (1997), 207238.

[11] D. Gabor, Theory of communication. J. Inst. Elec. Engr., 93 (1946), 429-457.

[12] S. Guliyev, Polar coordinates in Laguerre hypergroup. Khazar J. Math., 2 (2006), 11-20.

[13] I. S. Gradshteyn, I. M. Ryzhik, Table of Integrals. Series, and Products Seventh Edition, Elsevier Inc, 2007.

[14] G. H. Hardy, A theorem concerning Fourier transforms. J. London Math. Soc 8 (1933), 227-231

[15] V. Havin, B. Jöricke, The Uncertainty Principle in Harmonic Analysis. Springer, Berlin 1994.

[16] W. Heisenberg, Über den anschaulichen Inhalt der quantentheoretischen Kinematic und Mechanik. Zeit. Physik, 43, 172 (1927); The Physical Principles of the Quantum Theory (Dover, New York, 1949; The Univ. Chicago Press, 1930).

[17] J. Huang, H. Liu, A heat Kernel version of Hardy's theorem for the Laguerre Hypergroup. Acta. Math. Scientia. $30 \mathrm{~B}(2) 2010$.

[18] R. I. Jewett, Spaces with an abstract convolution of measures. Adv. Math., 18 (1973), 1-101.

[19] N. N. Lebedev, Special Functions and Thier Applications. Dover Publications, New York, 1972.

[20] M. M. Nessibi, K. Trimèche, Inversion of the Radon Transform on the Laguerre Hypergroup by using Generalized Wavelets. J. Math. Anal. Appl., 208 (1997), 337-363.

[21] M. M. Nessibi, B. Selmi, A Wiener-Tauberian and a Pompeiu type theorems on the Laguerre hypergroup. J. Math. Anal. Appl., 351 (2009), 232-243.

[22] J. F. Price, Inequalities and local uncertainty principles. J. Math. Phys., 24 (1983), 1711-1714.

[23] J. F. Price, Sharp local uncertainty principles. Studia Math., 85 (1987), 37-45.

[24] J. F. Price, A. Sitaram, Local uncertainty principles for locally compact groups. Trans. Amer. Math. Soc., 308 (1988), 105-114.

[25] M. Reed, B. Simon, Methods of Modern Mathematical Physics: Functional Analysis. Vol.1, Academic Press, 1980.

[26] M. Rösler, An uncertainty principle for the Dunkl transform. Bull. Austral Math. Soc. 59 (1999), 353-360.

[27] M. Rösler and M. Voit, An uncertainty principle for Hankel Transforms. Amer. Math. Society, 127 (1999), $183-$ 194.

[28] N. Shimeno, A note on the uncertainty principle for the Dunkl transform. J. Math. Sci. Univ. Tokyo, 8 (2001), 33-42.

[29] K. Stempak, An algebra associated with the generalized sublaplacian. Studia. Math., 88 (1988), 245-256.

[30] G. Szego, Orthogonal Ploynomials. Amer. Math. Society, Providence, RI, 1939.

[31] S. Thangavelu, Some uncertainty inequalities. Proc. Indian Acad. Sci. (Math. Sci.), 100 (1990), 137-145.

[32] K. Trimèche, Generalized wavelets and hypergroups. Gordon And Breach Science Publishers, 1997.

[33] N. Wiener, The Fourier Integral and Certain of its Applications. Cambridge, 1933.

[34] N. Wiener, I am a Mathematicia. Cambridge, 1956. 